\title{
Trust starts within
}

\author{
Dr. Katy Kamkar PhD, CPsych *
}

I am pleased to be taking on this new role as a Section Editor for this journal with a primary focus on ensuring our editorial content pays continuing attention to the broad issues of trust in relation to CSWB. There is so much good work that we will need to showcase and discuss with regard to this very human issue that underpins everything our CSWB professionals must do to serve our communities effectively. To open, though, I would like to focus some attention on our trust in, and our respect for, those professionals who serve across the many CSWB sectors.

Awareness and appreciation of mental health and well-being have increased in the past decade and more so in the past five years. We have also witnessed a reduction in stigma and change of attitudes related to mental illness. Increased communications and conversations, education, media, breakthrough research and evidence-based treatments, governments' new PTSD presumptive legislation, and collaboration and networking among other factors, have all contributed to the positive changes to date.

Improving and protecting the psychological health of individuals at work, as well as understanding the workplace and worker factors impacting health, are all part of workplace mental health. We have witnessed greater interest in advancing mental health promotion, prevention, and early intervention; and of identifying and boosting protective factors, reducing or eliminating risk factors, and building proactive strategies and resiliency.

Further training and education are needed to build positive culture, and for healthy supportive environments and interpersonal relationships. Moral injury, perception of injustice, burnout, compassion fatigue, lack of support, stigma and self-stigma can present barriers to recovery and prolong disability. Healthy interpersonal and communication skills and emotional intelligence need to be part of training and education for leaders-in particular, for all professionals working in human services. We all share the same human needs including the need for sense of safety, security, belonging, trust, autonomy, self-worth, independence, accomplishment, social justice, and self-efficacy. All those needs translate into our health and well-being-mental, physical, social, psychological and emotional health.

There is a need for the health care system, employers, workers, government, and compensation systems to engage in a collaborative approach to address the costs associated with mental health disability. The costs associated with mental health disabilities are higher than those of physicalrelated disabilities. The economic burden of mental illness in Canada is estimated at \$51 billion per year, and includes health care costs and lost productivity due to absenteeism and sick leave. We have increasingly seen that employers are faced with staggering disability and absenteeism rates due to mental illness, and that maintaining workplace mental health is an integral part of any business plan. Failing to have a comprehensive mental health strategy in the workplace contributes to long-term disability, unemployment, and family and financial strains. Research findings on the incidence and costs of physical and mental health-related disabilities highlight the importance of promoting mental health and well-being in the workplace, including among first responders and our police officers.

Police work does increase the risk of psychological work-related injuries. Police officers are exposed to a unique set of challenges in their day-to-day duties that can easily facilitate mental health concerns. Occupational burnout and exhaustion result in reduced motivation and care or passion for the work. For others, it can cause feelings of helplessness or powerlessness, resulting in emotional disengagement. Depression, substance misuse as a coping mechanism, and occupational stress injuries (OSI; which are persistent psychological difficulties resulting from operational or service-related duties) are also common. Depression has been found to increase the risk for post-traumatic stress disorder (PTSD), anxiety disorders and addiction. As well, PTSD has often been found to be accompanied by depression, and the two overlapping conditions further worsen the overall functioning and quality of life and increase the risk of suicide. Thus, the promotion of good mental health and mental health education, prevention, and early intervention, as well as the promotion of healthy and supportive work environments based on trust, support, and care, are essential to prevent complications resulting from concurrent issues. Ongoing work is needed in encouraging a proactive approach to health, and ensuring wellness programs include mental health and well-being.

Stigma still remains predominant in addition to other barriers to care and recovery that deserve further attention, research and/or interventions. Barriers include self-stigma, moral injury, burnout, perception of injustice, not recognizing that help is needed, fear of disclosure, inconsistent program application or administration, focus on crisis management rather than prevention, lack of awareness of program availability, difficulty accessing evidence-based treatments, delayed access to providers with expertise, as well as identifying and addressing organizational and individual stressors.

Correspondence to: Dr. Katy Kamkar, PhD, CPsych, Centre for Addiction and Mental Health (CAMH), Suite 200 - 455 Spadina Ave. Toronto, ON M5S 2G8. Tel: 416-535-8501 ext. 77337; Fax: 416-971-7172; E-mail: Katy.Kamkar@camh.ca 
Various strategies could be implemented to provide a supportive and safe work environment, including: providing supportive reintegration into the work environment after a leave of absence; providing stress management programs; aiming for work/life balance; encouraging use of health care professionals when someone is experiencing psychological distress; providing/confirming job security; having roles and responsibilities well-defined; having enough resources to cope with the demands of the job particularly during times of organizational changes and staff cutbacks that might result in more job demands and fewer resources; providing opportunities for growth and development; offering flexible work conditions whenever possible and appropriate; providing regular and constructive feedback and recognition for good performance; and providing healthy and supportive relationships and work environments that focus on trust, respect, support, gratitude, and care in the workplace.

This is why this topic must form part of our continuing dialogue on CSWB. If we do not attend to the stresses taken on by those whose work is directed to services, strengths, and justice for others, we will undermine the trust that is essential both within our human services systems, and among those systems and the public they are designed to serve.

CONFLICT OF INTEREST DISCLOSURES

The author declares there are no conflicts of interest.

AUTHOR AFFILIATIONS

*Centre for Addiction and Mental Health (CAMH), Toronto, ON. 